\title{
Traditional and Digital Biomarkers: Two Worlds Apart?
}

\author{
Lmar M. Babrak ${ }^{a} \quad$ Joseph Menetskib Michael Rebhan ${ }^{c}$ d \\ Giovanni Nisato $^{e}$ Marc Zinggeler $^{f}$ Noé Brasier $^{g}$ Katja Baerenfaller $^{h}$ \\ Thomas Brenzikofer $^{d} \quad$ Laurenz Baltzer ${ }^{i}$ Christian Vogler ${ }^{j} \quad$ Leo Gschwind $^{j}$

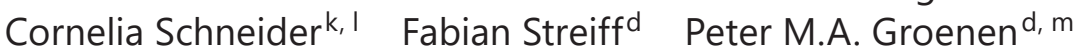 \\ Enkelejda Miho ${ }^{a}$ d, n
}

a FHNW University of Applied Sciences Northwestern Switzerland, Muttenz, Switzerland; ${ }^{\mathrm{b}}$ Foundation for the National Institutes of Health, North Bethesda, MD, USA; ${ }^{\mathrm{C}}$ Novartis Institutes for Biomedical Research, Basel, Switzerland; ${ }^{d}$ DayOne, BaselArea.Swiss, Basel,

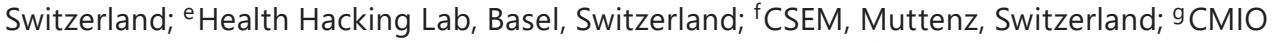

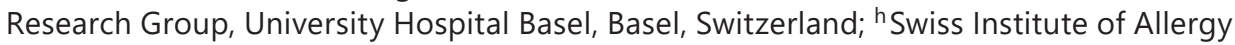
and Asthma Research (SIAF), University of Zurich, and Swiss Institute of Bioinformatics (SIB), Davos, Switzerland; 'S. Karger AG, Basel, Switzerland; 'Advancience AG, Allschwil, Switzerland; ${ }^{k}$ Clinical Pharmacy and Epidemiology, Department of Pharmaceutical Sciences, University of Basel, Basel, Switzerland; 'Hospital Pharmacy, University Hospital Basel, Basel,

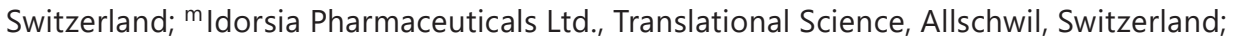
naiNET GmbH, Basel, Switzerland

\section{Keywords}

Traditional biomarkers · Digital biomarkers · Semantics · Biomarker classification · Digital health

\section{Abstract}

The identification and application of biomarkers in the clinical and medical fields has an enormous impact on society. The increase of digital devices and the rise in popularity of healthrelated mobile apps has produced a new trove of biomarkers in large, diverse, and complex data. However, the unclear definition of digital biomarkers, population groups, and their intersection with traditional biomarkers hinders their discovery and validation. We have identified current issues in the field of digital biomarkers and put forth suggestions to address them during the DayOne Workshop with participants from academia and industry. We have found similarities and differences between traditional and digital biomarkers in order to synchronize semantics, define unique features, review current regulatory procedures, and describe novel applications that enable precision medicine.

Enkelejda Miho

FHNW University of Applied Sciences Northwestern Switzerland

Hofackerstrasse 30

$\mathrm{CH}-4132$ Muttenz (Switzerland)

E-Mail enkelejda.miho@fhnw.ch
Peter Groenen

Idorsia Pharmaceuticals Ltd

Hegenheimermattweg 91

CH-4123 Allschwil (Switzerland)

E-Mail peter.groenen @idorsia.com 
Babrak et al.: Traditional versus Digital Biomarkers

\section{Introduction}

Biomarkers are an integral part of biomedical research and clinical practice. Many common research assays and clinical tests, such as measuring cholesterol levels, blood pressure, body temperature, or pulmonary function tests serve as biomarkers for assessing health states. In clinical trials, well-defined traditional biomarkers are important and accepted metrics to objectively assess clinical status, therapeutic effects such as early predictors for clinical endpoints, and adverse events. Due to the globally increasing importance of biomarkers in modern healthcare, in 1998 the US National Institute of Health (NIH) Biomarkers Definitions Working Group began to define clinically associated terms and opinions on the qualification of novel methodologies for medicine development. Since, they have also been adopted by the European Medicines Agency (EMA) Committee for Medicinal Products for Human Use (CHMP) [1]. A biomarker is defined as "a characteristic that is measured as an indicator of normal biological processes, pathogenic processes, or responses to an exposure or intervention including therapeutic interventions. This can include molecular, histological, radiographic, or physiologic characteristics" [2].

The FDA-NIH Joint Leadership Council put together the Biomarkers, Endpoints and other Tools Resource (BEST) in order to harmonize translational science by standardizing medical terms and improve scientific understanding regarding biomarkers in clinical development and practice [2]. In BEST, the FDA-NIH Biomarker Working Group defined two categories of biomarkers: (i) disease-associated biomarkers (susceptibility/risk biomarker, diagnostic biomarker, prognostic biomarker, monitoring biomarker), and (ii) drug-related biomarkers (predictive biomarker, pharmacodynamics/response biomarker, safety biomarker). For example, body mass index (BMI) measurement is a risk biomarker used to assess the risk for metabolic diseases such as diabetes mellitus, hypertension, and dyslipidemia, and can lead to preventative health measures [3]. Biomarkers can also be safety indicators for determining health status, such as liver function, by measuring serum creatinine, blood urea nitrogen, and cystatin $C[4,5]$. The working group also describes other clearly standardized biomarkerrelevant definitions, e.g., "fit for purpose" and "context of use (COU)" [2].

Due to the longand resource-intensive process, incorporation of fully qualified biomarkers in the medical field is very challenging. It begins with biomarker discovery, animal experiments, clinical or epidemiological studies, analytical validation, and interventional studies with the gold standard endpoints [6-8]. Additionally, there are many stakeholders involved in the qualification of biomarkers: academia, technology companies, biopharmaceutical industry, regulatory agencies, doctors, and patients add to the complexity of biomarker qualification and acceptance.

As digital devices have begun to be integrated into the medical landscape, digital biomarkers have become an exciting new tool for advancing precision medicine and supporting clinical trials. Digital biomarkers are objective, quantifiable, physiological, and behavioral measures that are collected by means of digital devices that are portable, wearable, implantable, or digestible [9]. These data are often used to explain, influence, and/or predict health-related outcomes. Individualized measures can now be recorded to create personalized baselines for health. The inclusion of digital biomarkers has specifically impacted the field of neurology where there is a great unmet need for objective and non-invasive biomarkers. Currently, a number of digital biomarkers are being tested for feasibility and reliability in Parkinson's and Alzheimer's disease and clinical outcome assessments [10,11].

As the emerging field of digital biomarkers joins traditional biomarkers in the health(care) innovation process [12], it is crucial to address rising new possibilities and challenges, but most importantly define a standardized nomenclature and process to allow for a more rapid clinical uptake. To tackle this pressing need, the BaselArea.Swiss organized the DayOne 
Babrak et al.: Traditional versus Digital Biomarkers

Fig. 1. Digital technologies have enabled the measurement of digital biomarkers. Traditional biomarkers are divided into seven categories depending on the clinical goal for the biomarker (i.e., safety, predictive, diagnostic, etc.). We propose that digital technologies enable the measurement of digital biomarkers, which contribute new and unique features (e.g., longitudinal, continuous measurements) while sharing the same traditional biomarker clinical goals.

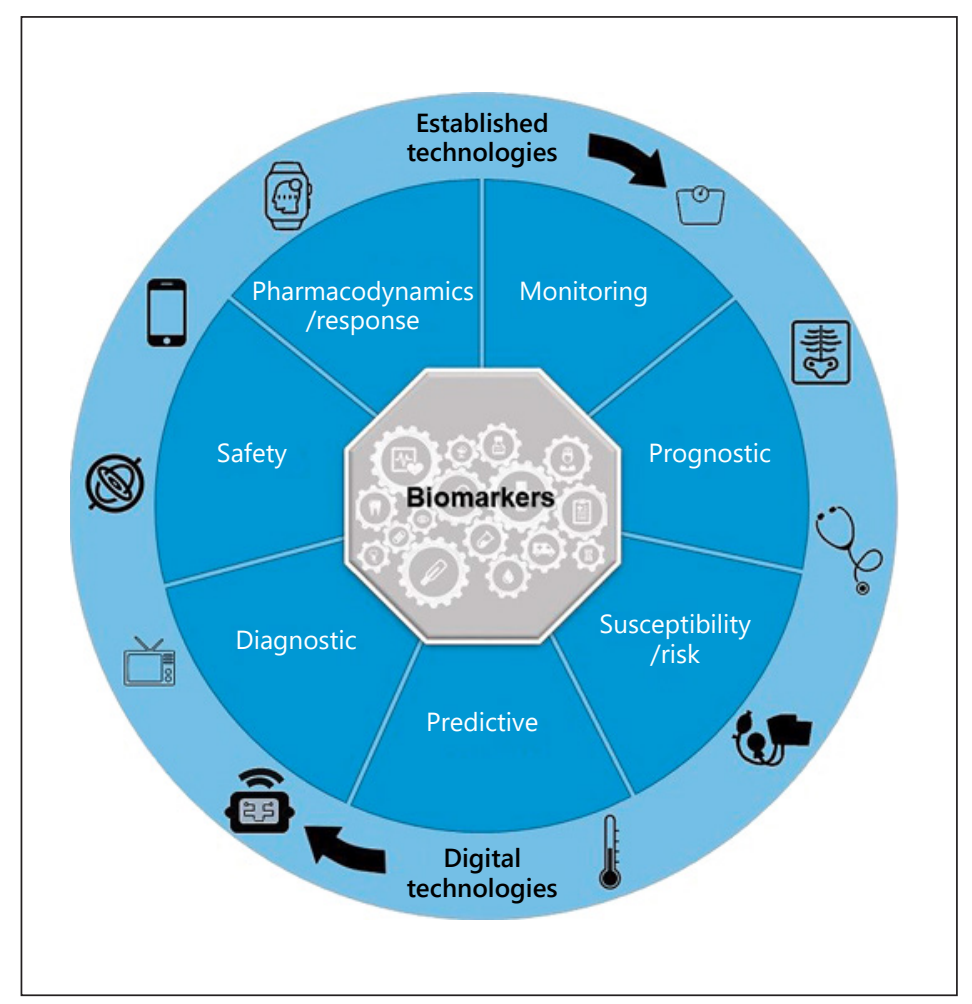

Workshop "Traditional and Digital Biomarkers - Two Worlds Apart" on March 27, 2019 in Basel, Switzerland. A diverse and relevant group of stakeholders from academia and industry attended, bringing together multiple viewpoints. In this perspective, we present our findings on identifying similarities and differences between traditional and digital biomarkers and a discussion on how these fields could be further harmonized by addressing semantics, features unique to each area, and novel applications in the medical field.

\section{A Comparison of Traditional and Digital Biomarker Characteristics}

Despite the apparent similarities between traditional and digital biomarkers in the way they address important challenges related to the health-disease continuum, there are also important differences in culture, innovation, scientific and technical maturity, and the nature of the data. Simply, digital biomarkers fall within the scope of traditional biomarkers in relation to addressing health-related questions, with use of a digital and portable technology that adds new dimensions, unique features and challenges (Fig. 1; Tables 1,2).

Traditional biomarkers are often well embedded into clinical practice and research, usually in proximity to the pathological event of interest. They are generally limited in analytical complexity and can range from being qualitative to quantitative. However, such traditional biomarkers can be invasive and expensive to measure. Due to the dynamic, complex nature of disease, traditional biomarkers often present an incomplete view due to the limited number of measurements that can be collected over time ("snapshot" problem). Alternatively, digital biomarkers are usually less or non-invasive, modular, and often cheaper to measure. They can produce qualitative and quantitative measurements, but most importantly, they provide easier and cheaper access to continuous and longitudinal measurements. Nonetheless, digital biomarkers are still new and therefore are not commonly implemented 
Babrak et al.: Traditional versus Digital Biomarkers

Table 1. Overview of traditional and digital biomarker features, novel applications, and published examples

\section{Biomarkers}

Definition: "a characteristic that is measured as an indicator of normal biological or pathogenic processes, or responses to an exposure or intervention" [2]

\begin{tabular}{|c|c|c|}
\hline $\begin{array}{l}\text { Feature in digital } \\
\text { biomarkers }\end{array}$ & Novel applications & Examples \\
\hline $\begin{array}{l}\text { Individual/ } \\
\text { population } \\
\text { level health data }\end{array}$ & $\begin{array}{l}\text { Clinical trial patient stratification } \\
\text { Clinical trial patient recruitment } \\
\text { Development of personalized/population } \\
\text { baselines } \\
\text { Population health management }\end{array}$ & $\begin{array}{l}\text { Detection risk of dementia with computerized cognitive } \\
\text { testing [13] } \\
\text { Wearable sensors to monitor gait performance in } \\
\text { Huntington disease (recorded }>14,000 \text { assessments vs. } 20 \text { in } \\
\text { the clinic) [14] }\end{array}$ \\
\hline $\begin{array}{l}\text { Longitudinal and } \\
\text { continuous } \\
\text { measurements }\end{array}$ & $\begin{array}{l}\text { More data points will lead to higher } \\
\text { granularity and stronger analysis } \\
\text { Clearer understanding of health status } \\
\text { High resolution of data to stratify } \\
\text { subgroups within a population } \\
\text { Measures change over time } \\
\text { Serves as a control for disease states } \\
\text { Measure/observe episodic medical } \\
\text { occurrences in real-time, outside of clinic }\end{array}$ & $\begin{array}{l}\text { Analysis of finger tapping and memory tests conducted on } \\
\text { smartphones for characterization of longitudinal } \\
\text { characteristics of Parkinson's disease [15] } \\
\text { Voice, finger tapping, gait, and reaction time on smartphone } \\
\text { application for developing an objective measure of PD } \\
\text { severity [16] } \\
\text { Wearable devices for real-time detection of epileptic seizures } \\
\text { to better understand the condition and option to contact } \\
\text { emergency services [17] }\end{array}$ \\
\hline $\begin{array}{l}\text { Passive } \\
\text { monitoring }\end{array}$ & $\begin{array}{l}\text { Facilitates monitoring outside of hospital } \\
\text { Objective data (individual-independent) } \\
\text { Low patient burden; higher adherence }\end{array}$ & $\begin{array}{l}\text { Automated analysis of free speech to predict psychosis onset } \\
\text { in youths [18] } \\
\text { Passively acquired accelerometer data as primary endpoint } \\
\text { to measure the effect of isosorbide mononitrate treatment in } \\
\text { patients with heart failure [19] }\end{array}$ \\
\hline
\end{tabular}

Table 2. Current challenges of digital biomarkers in medical settings

\begin{tabular}{ll}
\hline Challenges & Explanation \\
\hline Semantics & $\begin{array}{l}\text { Lack of standardization in the choice of words describing novel concepts in digital biomarkers } \\
\text { (e.g., longitudinal data) }\end{array}$ \\
\hline $\begin{array}{l}\text { Data standardization } \\
\text { Large number of heterogeneous data sources, formats, scales } \\
\text { of user data, anonymization }\end{array}$ & $\begin{array}{l}\text { Regulatory guidelines on privacy, right to opt out, informed consent, data ownership is not fully } \\
\text { developed, continuously changing, and different between countries }\end{array}$ \\
\hline $\begin{array}{l}\text { Data storage } \\
\text { Large volumes of complex data are being generated but there are few guidelines on how they } \\
\text { should be stored }\end{array}$ \\
\hline $\begin{array}{l}\text { Identification of relevant } \\
\text { data and interpretation }\end{array}$ & $\begin{array}{l}\text { Identification of pertinent data is important for analysis, and how to correctly analyze the data } \\
\text { and use accurate baselines is still unclear; this process is often not transparent due to } \\
\text { proprietary issues }\end{array}$ \\
\hline $\begin{array}{l}\text { Regulatory approval: data } \\
\text { analytics, algorithm and tool } \\
\text { validation }\end{array}$ & $\begin{array}{l}\text { Regulatory standards lag behind rapid innovation in tool, analytics, and algorithm development } \\
\end{array}$ \\
\hline
\end{tabular}

in clinical development and practice. Ease-of-use and low cost come to the detriment of often measuring distally from pathological events, and producing large, complex data that are challenging for data analytics. Some additional drawbacks include data integrity assessment and reliability, hardware malfunctions, identification of relevant data, multiple interpretations, and baseline determination. 
Babrak et al.: Traditional versus Digital Biomarkers

\section{Standardizing Novel Semantics Is a Challenge}

In any emerging field, a high degree of ambiguity in semantics is typical. In digital biomarkers this results from the merging of several fields (healthcare, engineering, and analytics), from the commercial marketing of mobile products directly to the public, and from a lack of shared practices and standardization. Further, proprietary devices and corresponding digital biomarkers are introduced in the market with differing terminologies used for medical, scientific, and marketing purposes. For example, gait - the way an individual walks - is termed and described differently depending on the context (e.g., medical, engineering, analytics) and would be described differently for marketing purposes depending on the target demographic group. Adding to this disparity, there is also a cultural and historical ambiguity in terminology. There are terms in the regulatory space that have legacy connotations that may no longer apply, or are co-opted or newly created without sufficient standardization and consensus among participating stakeholders. These semantic issues generate confusion by creating conflicting or divergent terms. While a certain degree of semantic ambiguity is present, we propose using traditional biomarker semantics to describe digital biomarkers. While terms such as "surrogate endpoint," and "validation" can apply to both types of biomarkers, additional concepts such as continuous and longitudinal data must be defined to encompass these new parameters and how these terms relate to each other.

Community-based semantic disambiguation in emerging areas is no trivial pursuit and requires an agile, iterative process of refining and shaping terms that enables the community to focus on areas that need our attention first, considering the costs of non-action. For these new terms to be incorporated and modified, a living and dynamic standard for terms needs to be assembled and maintained. This allows new terms to be integrated while removing outdated, irrelevant terms. This living dictionary will need to be flexible, keep up with the rapid speed of technological innovation, and reflect community decisions by expert decisionmakers, producers, and users of data, and be based in the regulated field of patient healthcare.

\section{Digital Biomarker Users Consist of a Diverse Population}

While traditional biomarkers are applied to a population group seeking to improve or assess their health status, the digital biomarker application landscape is broader. It encompasses three different population groups: (i) the casual, curiosity, or fitness-based individual for non-clinical use (often in the healthier section of the health-disease continuum), (ii) the commercial-based group, and (iii) the regulatory and clinical trial group (typically in the more diseased cluster of the health-disease continuum; Fig. 2). To fully understand and address the challenges in the field of digital biomarkers, the diversity of the population groups that produce these data needs to be identified and attributes described. Figure 1 depicts three different population groups and highlights several unique characteristics within these groups. The first group falls into the casual non-clinical use of digital biomarkers, which includes those who are curious, fitness-trackers, the "quantified self," and individual-driven phenotyping or status tracking. This population group consists of the largest number of individuals, but contains the least amount of reliable, validated, and consistent data, and is at a higher risk of data breach [20,21]. These attributes prevent the use of this type of data in a medical context. Technological industries associated with this group typically change products rapidly, preventing them from complying with complex regulatory settings which would normally slow down the ability to adapt products quickly to a rapidly changing market.

The second population consists of users of digital biomarkers for commercial purposes such as fitness coaching for professional athletes and for facilitating decision-making in 


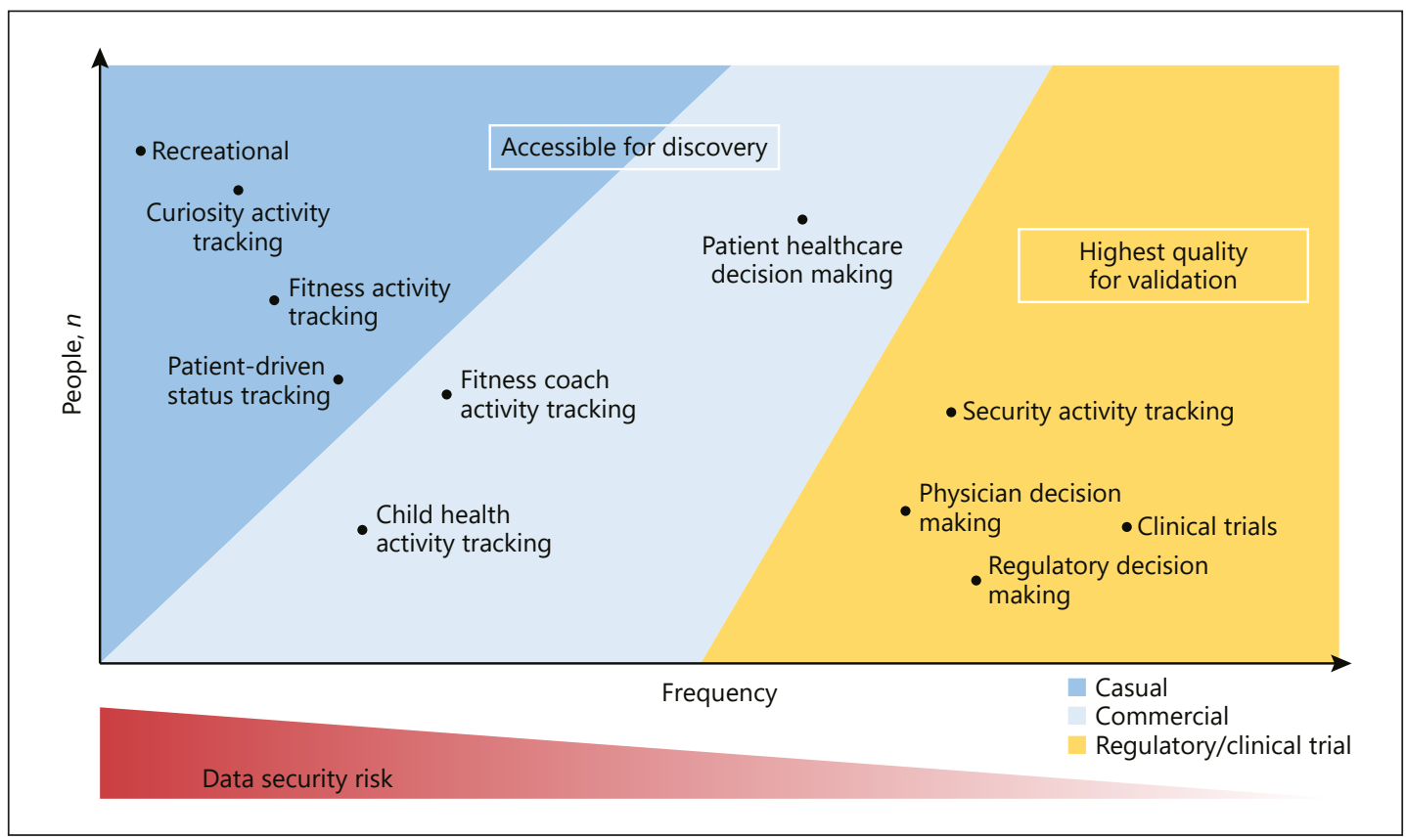

Fig. 2. User populations. Different populations of data producers of potential digital biomarkers and device users are grouped based on application, size of population, consistency of measure, and data security risk, and are categorized based on the best potential use of the data for discovery of novel digital biomarkers or validation. The $y$-axis describes the number of individuals measured and the $x$-axis describes the consistency of the measure. Consistency is defined as accuracy, precision, reproducibility, calibration, and traceability.

healthcare. These users are often located somewhere in the center of the health-disease continuum. This group is highly variable in size but is generally smaller than the first population group. For example, ORECCO, a privately-owned company, provides professional athletes with performance results based on the digital biomarkers they collect [22].

The third population group consists of the device users and data producers involved with clinical or regulatory decision-makers, pharmaceutical companies, medtech, or physicians. This population group is the smallest and the security against improper alteration of the data needs to be high due to the medical and regulatory implications. The consistency and quality of the data (i.e., accuracy, precision, reproducibility, calibration, and traceability) is the highest, containing strict definitions, protocols, and predetermined analyses, making this group ideal for digital biomarker validation. In this group, physicians specialized in particular diseases typically recruit participants according to specific inclusion and exclusion criteria, and traditionally no information optimized for interpretation by patients goes back to the patients.

Despite their differences, the three population groups can provide valuable information with regard to population baselines and health status (casual and commercial groups), which can be formally implemented for biomarker validation (regulatory/clinical study group). In the digital biomarker landscape, the number of individuals measured, data security, and consistency of data vary greatly. Due to the diversity of features demonstrated by each population group depicted in Figure 2, there is an expected level of confusion and uncertainty regarding how to define terms and apply regulatory applications. We need to recognize that there is a breadth of digital biomarker applications and each should be defined separately, standardized appropriately, and regulated accordingly depending on the population group. 
Babrak et al.: Traditional versus Digital Biomarkers

Fig. 3. Biomarker classification. Both traditional and digital biomarkers can be classified based on the status of a particular measurement to a particular clinical status or outcome. A digital biomarker either replaces a non-digital biomarker (Approved), opens a new field (Novel) or is a hybrid that on the one hand replaces and on the other hand opens a new field (Original).

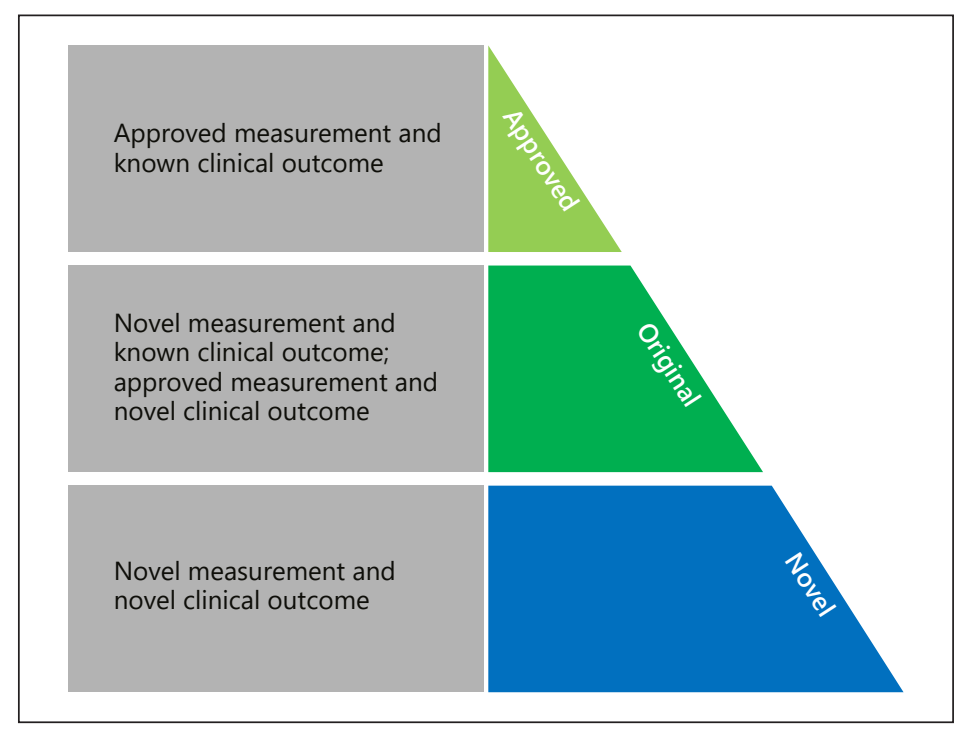

\section{The Classification of Digital Biomarkers}

Although there are many perspectives on how digital technologies can be classified (i.e., clinical outcomes or biomarkers), we set to further clarify and describe digital biomarkers in the scope of clinical development and practice. We propose a method of classifying digital biomarkers by taking into account the digital measurement tool and clinical outcome assessment (COA; Fig. 3, adapted from [23]). COA is the monitoring of how a patient feels, functions, or survives. An important corollary to the COA definition is that a biomarker is not a measurement of how a patient feels, functions, or survives. Digital biomarkers and their associated clinical assessment outcomes are partitioned into three types. "Approved" describes biomarkers that improve or accelerate generally accepted practices. These are digital biomarkers that consist of approved measurements such as heart rate, pulse, and known clinical outcomes, such as cardiac risk [24]. "Original" characterizes biomarkers from two scenarios: (i) a novel measurement with a known clinical outcome such as gait measurement for Parkinson's disease assessment [10] and (ii) an approved measurement, such as heart rate to describe a novel clinical outcome such as depression [25]. "Novel" describes a novel measurement such as physical activity or facial expressions, with a novel clinical outcome such as depression [26]. "Approved" digital biomarkers will likely be the first ones to be used as they are already medically validated and implemented in the clinic. Digital biomarkers in "Original," and especially in "Novel," will require rigorous testing and validation to become adopted in clinical development. This new classification system will help facilitate and clarify the type of biomarker and clinical outcome being described and facilitate regulations.

\section{Regulatory Concepts in Digital Biomarkers}

The regulatory procedures concerning biomarkers, including digital, is complex because they can be applied to a broad spectrum of uses such as drugs/biologicals and medical devices, and regulated differently in various countries. In Switzerland, the council directive concerning medical devices 93/42/EEC (Swiss Agency for Therapeutic Products, Swissmedic) defines 
"medical device" broadly as any instrument, apparatus, appliance, software, material, or other article, whether used alone or in combination, used specifically for diagnostic and/or therapeutic purposes. In the USA, the Food and Drug Administration (FDA) oversees the Center for Drug Evaluation and Research (CDER), the Center for Biologics Evaluation and Research (CBER), and the Center for Devices and Radiological Health (CDRH), which manage the approval of new drugs, biologics, and medical devices, respectively. Currently in Europe, the European Medicines Agency (EMA) manages the approval of medical devices and drugs, but recently, a new legislation has created the Medical Device Regulation (MDR), which will oversee all new medical devices and enforce more stringent rules compared to the CDRH for medical device approval in 2020 [27]. Generally, in the USA, medical devices with software that operates the device and sensors have been categorized as "software in medical device" (SiMD). With the introduction of digital biomarkers, new terminology was introduced: "software as a medical device" (SaMD). SaMD is defined as software that performs a medical function without being part of the hardware (e.g., machine learning tools in mobile apps) [28]. An example of an SaMD is the Apple Watch software for atrial fibrillation detection where the Apple Watch serves a component supporting digital biomarker measurements (atrial fibrillation).

For drug and biological measurements and approvals, digital tools are being adopted in the first three phases of clinical trials, and the data derived need to be collected early and consistently to develop the necessary evidence for internal decision-making (phase I and II clinical trials) and regulatory approval (phase III) [28]. In the regulatory area, tools and measurements for medical product development are termed drug development tools or medical device development tools. The FDA also has a Prescription Drug-Use-Related Software that is developed for use with prescription drugs (e.g., drug ingestion tracking, dose calculation, remainders, and drug instructions). Similar to the drug-related products described above, medical devices go through a process for clearance or approval [28]. In this process, the technical aspects of the design of the product are more carefully considered. The intended use of the device determines if and how the item is regulated. If the medical device is only intended to be used for wellness purposes then no regulation is necessary, but if the same device claims to diagnose or monitor a health condition, it needs to be regulated. Whether software is considered a device is determined by a regulatory body and will be highly dependent on the intended functions. The FDA continues to update guidelines in order to clearly determine which devices should be regulated, but the distinctions of what is considered a medical device is still unclear, and often exceptions are made.

\section{Novel Applications for the Fields of Precision Medicine}

Digital biomarkers are also extremely well suited for applications and medical areas that rely on subjective measures such as neurology and psychiatry. Digital biomarkers can provide objective measurements to base and support diagnosis, prognosis, and measure therapeutic outcomes. In fields like Alzheimer's and Parkinson's disease, digital outcome measurements bring a paradigm shift of how treatment outcomes are measured and assessed, particularly in very early (prodromal) disease states [29]. Currently, many pharmaceutical companies are running pilot studies to test the feasibility of using digital biomarkers. For example, Roche has built a Parkinson's disease app to measure active and passive PD tests and assess disease severity [30], Biogen is in partnership with PatientsLikeMe trying to understand physical activity measurements in patients with multiple sclerosis [31], and Neurotrack has remotely measured cognitive ability to assess cognition in patients with Alzheimer's disease [32]. In psychiatry, using "digital behavioral biomarkers" such as phone usage patterns in health 
Babrak et al.: Traditional versus Digital Biomarkers

disorders has had encouraging results [33]. Additionally, digital biomarkers are predictive for the diagnosis and prognosis of symptom severity in patients with major depressive or bipolar disorders [34]. Unfortunately, there are few published results and currently it is difficult to assess the overall feasibility, technology and biology behind digital biomarkers.

\section{Conclusion}

Digital continuous monitoring has brought a wealth of new data to healthcare. Retrospectively in science, there have been many instances where new technology has propelled a paradigm shift that enabled new questions and novel insights. Digital biomarkers may not shift the medical paradigm directly, but these novel ways of measuring health status provide observations and perspectives into disease that were unavailable before. They supplement and enhance conclusions from traditional biomarkers (e.g., dynamic changes measured with digital biomarkers during the course of a disease). Detailed longitudinal measurements, combined with accurate and precise assessments from molecular characterization of health and disease, have the potential to redefine diagnosis and the medical classification of diseases. Similar to novel stratifications in cancer groups due to molecular profiling, digital biomarkers can further support precision medicine and lead to innovative treatments. Lastly, digital biomarkers provide information that has the potential to greatly influence not only what we know about disease and prevention, but our very understanding of health.

\section{Acknowledgements}

We would like to acknowledge the DayOne community.

\section{Disclosure Statement}

J.M. declares holding shares in Merck Pharmaceuticals (MSD outside the USA). P.M.A.G. declares holding shares in Idorsia Pharmaceuticals. E.M. declares holding shares in aiNET GmbH. L.B. is a publishing manager at Karger AG. The remaining authors declare no competing interests.

\section{Funding Sources}

Authors are grateful to FHWN University of Applied Sciences and Arts Northwestern Switzerland and BaselArea.Swiss for funding this research.

\section{Author Contributions}

All authors contributed to the discussions and wrote the manuscript. L.M.B, J.M., and E.M. produced the figures. 


\begin{tabular}{|c|c|}
\hline \multicolumn{2}{|l|}{ Digit Biomark 2019;3:92-102 } \\
\hline DOI: 10.1159/000502000 & $\begin{array}{l}\text { (c) } 2019 \text { The Author(s). Published by S. Karger AG, Basel } \\
\text { www.karger.com/dib }\end{array}$ \\
\hline
\end{tabular}

Babrak et al.: Traditional versus Digital Biomarkers

\section{References}

1 European Medicines Agency, Committee for Medicinal Products Human Use (CHMP)European Medicines Agency. (2018) (available at https://www.ema.europa.eu/en/committees/committee-medicinalproducts-human-use-chmp)

2 FDA-NIH Biomarker Working Group. BEST (biomarkers, endpoints, and other tools) resource. Silver Spring: FDA; 2016. Available from: http://www.ncbi.nlm.nih.gov/books/NBK326791/

3 Bays HE, Chapman RH, Grandy S; SHIELD Investigators' Group. The relationship of body mass index to diabetes mellitus, hypertension and dyslipidaemia: comparison of data from two national surveys. Int J Clin Pract. 2007 May;61(5):737-47.

4 Kar S, Paglialunga S, Islam R. Cystatin C is a more reliable biomarker for determining eGFR to support drug development studies. J Clin Pharmacol. 2018 Oct;58(10):1239-47.

5 Bonventre JV, Vaidya VS, Schmouder R, Feig P, Dieterle F. Next-generation biomarkers for detecting kidney toxicity. Nat Biotechnol. 2010 May;28(5):436-40.

6 Kraus VB. Biomarkers as drug development tools: discovery, validation, qualification and use. Nat Rev Rheumatol. 2018 Jun;14(6):354-62.

7 Allinson JL. Clinical biomarker validation. Bioanalysis. 2018 Jun;10(12):957-68.

8 Hayes DF. Biomarker validation and testing. Mol Oncol. 2015 May;9(5):960-6.

9 Dorsey ER, editor. Digital biomarkers. Basel: Karger. Available from: https://www.karger.com/Journal/ Home/271954

10 Lipsmeier F, Taylor KI, Kilchenmann T, Wolf D, Scotland A, Schjodt-Eriksen J, et al. Evaluation of smartphonebased testing to generate exploratory outcome measures in a phase 1 Parkinson's disease clinical trial. Mov Disord. 2018 Aug;33(8):1287-97.

11 Kourtis LC, Regele OB, Wright JM, Jones GB. Digital biomarkers for Alzheimer's disease: the mobile/wearable devices opportunity. NPJ Digit Med. 2019;2:9.

12 Wright JM, Jones GB. Harnessing the Digital Exhaust: incorporating wellness into the pharma model. Digit Biomark. 2018 Jan-Apr;2(1):31-46.

13 Ritchie K, Carrière I, Su L, O'Brien JT, Lovestone S, Wells K, et al. The midlife cognitive profiles of adults at high risk of late-onset Alzheimer's disease: the PREVENT study. Alzheimers Dement. 2017 Oct;13(10):1089-97.

14 Andrzejewski KL, Dowling AV, Stamler D, Felong TJ, Harris DA, Wong C, et al. Wearable Sensors in Huntington Disease: A Pilot Study. J Huntingtons Dis. 2016 Jun;5(2):199-206.

15 Prince J, Arora S, de Vos M. Big data in Parkinson's disease: using smartphones to remotely detect longitudinal disease phenotypes. Physiol Meas. 2018 Apr;39(4):044005.

16 Zhan A, Mohan S, Tarolli C, Schneider RB, Adams JL, Sharma S, et al. Using Smartphones and Machine Learning to Quantify Parkinson Disease Severity: The Mobile Parkinson Disease Score. JAMA Neurol. 2018 Jul;75(7):876-80.

17 Bruno E, Simblett S, Lang A, Biondi A, Odoi C, Schulze-Bonhage A, et al. Wearable technology in epilepsy: the views of patients, caregivers, and healthcare professionals. Epilepsy Behav. 2018 Aug;85:141-9.

18 Bedi G, Carrillo F, Cecchi GA, Slezak DF, Sigman M, Mota NB, Ribeiro S, Javitt DC, Copelli M, Corcoran CM. Automated analysis of free speech predicts psychosis onset in high-risk youths. NPJ Schizophr. 2015;1:15030.

19 Redfield MM, Anstrom KJ, Levine JA, Koepp GA, Borlaug BA, Chen HH, et al.; NHLBI Heart Failure Clinical Research Network. Isosorbide Mononitrate in Heart Failure with Preserved Ejection Fraction. N Engl J Med. 2015 Dec;373(24):2314-24.

20 Case MA, Burwick HA, Volpp KG, Patel MS. Accuracy of smartphone applications and wearable devices for tracking physical activity data. JAMA. 2015 Feb;313(6):625-6.

21 Kooiman TJ, Dontje ML, Sprenger SR, Krijnen WP, van der Schans CP, de Groot M. Reliability and validity of ten consumer activity trackers. BMC Sports Sci Med Rehabil. 2015 Oct;7(1):24.

22 Orreco. Data driven solutions for optimizing athlete performance. Galway: Orreco; 2019. Available from: https://orreco.com/

23 Wang T, Azad T, Rajan R. The emerging influence of digital biomarkers on healthcare. San Francisco: Rock Health; 2019. Available from: https://rockhealth.com/reports/the-emerging-influence-of-digitalbiomarkers-on-healthcare/

24 Perret-Guillaume C, Joly L, Benetos A. Heart rate as a risk factor for cardiovascular disease. Prog Cardiovasc Dis. 2009 Jul-Aug;52(1):6-10.

25 Carney RM, Freedland KE. Depression and heart rate variability in patients with coronary heart disease. Cleve Clin J Med. 2009 Apr; 76(suppl 2):S13-7.

26 Girard JM, Cohn JF, Mahoor MH, Mavadati S, Rosenwald DP. Social Risk and Depression: Evidence from Manual and Automatic Facial Expression Analysis, Proc Int Conf Autom Face Gesture Recognit, 1-8 (2013).

27 Swissmedic. Information on the EU regulations (MDR, IVDR). Bern: Swissmedic; 2019. Available from: https:// www.swissmedic.ch/swissmedic/en/home/medizinprodukte/neue-eu-verordnungen-mdr-ivdr-/aktuelleszur-umsetzung.html

28 Coravos A, Goldsack JC, Karlin DR, Nebeker C, Perakslis E, Zimmerman N, et al. Digital medicine: a primer on measurement. Digit Biomark. 2019;3:31-71.

29 Gold M, Amatniek J, Carrillo MC, Cedarbaum JM, Hendrix JA, Miller BB, et al. Digital technologies as biomarkers, clinical outcomes assessment, and recruitment tools in Alzheimer's disease clinical trials. Alzheimers Dement. 2018 May; 4:234-42. 
Babrak et al.: Traditional versus Digital Biomarkers

30 Roche. Roche technology measures Parkinson's disease fluctuations. Basel: Roche; 2019. Available from: https://www.roche.com/media/store/roche_stories/roche-stories-2015-08-10.htm

31 Moore C. PatientsLikeMe/Biogen study evaluates wearable devices to monitors activity of people with MS. Philadelphia: Multiple Sclerosis News Today; 2015. Available from: https://multiplesclerosisnewstoday. com/multiple-sclerosis-news/2015/04/21/patientslikemebiogen-study-evaluates-wearable-devices-monitors-activity-people-ms /

32 Neurotrack [Internet]. Redwood City: Neurotrack; 2019. Available from: https://www.neurotrack.com/ science/

33 Faurholt-Jepsen M, Vinberg M, Frost M, Debel S, Margrethe Christensen E, Bardram JE, et al. Behavioral activities collected through smartphones and the association with illness activity in bipolar disorder. Int J Methods Psychiatr Res. 2016 Dec;25(4):309-23.

34 Jacobson NC, Weingarden H, Wilhelm S. Digital biomarkers of mood disorders and symptom change. NPJ Digit Med. 2019;2:3. 